\title{
Breaking Down the Barriers in Complicated Pleural Sepsis
}

\author{
Morné J. Vorster Coenraad F.N. Koegelenberg \\ Division of Pulmonology, Department of Medicine, Stellenbosch University and Tygerberg Academic Hospital, \\ Cape Town, South Africa
}

'If a pleurisy lasts 20 days then it forms an empyema and if it does not rupture then death will occur.' Although Hippocrates described the natural course of empyema more than two millenniums ago and despite the fact that our knowledge of medicine has increased exponentially during the last few decades, complicated pleural infections still carry up to a 20\% 1-year mortality in the 21 st century [1].

The development of a parapneumonic effusion occurs in three clinically relevant stages that represent a continuum. A rapid influx of exudative fluid into the pleural space is observed in up to $40 \%$ of patients with pneumonia and heralds the first or exudative stage [1]. The accumulation of fluid is due to increased pulmonary interstitial fluid traversing the pleura to enter the pleural space and an increase in vascular permeability secondary to pro-inflammatory cytokines. Most patients with uncomplicated parapneumonic effusions will respond to antibiotics alone and drainage is generally not required. Untreated exudative effusions may develop into fibrinopurulent effusions. During the fibrinopurulent stage, the pleural space becomes increasingly infected. Loculations may develop and closed or open drainage becomes necessary, the point in time where an effusion is referred to as 'complicated' [1]. A critical characteristic of the fibrinopurulent stage of pleural sepsis is the disturbance of the physiological equilibrium between clotting and fibrinoly-

\section{KARGER}

E-Mail karger@karger.com

www.karger.com/res sis within the pleural space. Increased procoagulant and decreased fibrinolytic activity in response to infection is unique to the serosal spaces and although the exact mechanism still needs to be elucidated, its effects are well known: pleural surfaces coated with fibrin and fibrin strands with secondary adhesions and loculations, all complicating pleural fluid drainage [1]. The third and final stage of pleural infection is the organising phase.

The mainstay of treatment of complicated parapneumonic effusions remains drainage of the infected pleural fluid with chest tube thoracostomy and appropriate antibiotic cover. This course of management is frequently unsuccessful, which traditionally necessitated referral for surgical interventions (thoracotomy or thoracoscopy). Conservative management tends to fail in elderly or those with multiple comorbidities, which not infrequently preclude them from surgery. The reasons for failing conservative management seem to stem directly from the characteristics of the infected pleural space, with high fluid viscosity and fibrous septations impeding drainage [1]. These characteristics have become the targets of medical management aimed at improving the outcome of pleural infections and avoiding referral for surgery.

Tillett and Sherry [2] were responsible for the introduction of intrapleural fibrinolysis as a supplement to antimicrobial therapy as early as 1949. Although the theoretical advantage of fibrinolytics certainly seemed obvious, it took
(C) 2016 S. Karger AG Basel

0025-7931/16/0912-0099\$39.50/0 
many decades before a firm evidence base could be established to support the use of this and related modalities.

Diacon et al. [3] showed that intrapleural streptokinase, aimed at fibrinolysis of septations, could reduce surgical referrals. These results could not be replicated by Maskell et al. [4] in the much larger Multicenter Intrapleural Sepsis Trial (MIST)-1, comparing intrapleural streptokinase with placebo and showing no difference in outcome. Some limitations of the MIST-1 trial included the omission to report the volume of fluid drained and the extensive use of small-bore chest tubes [5].

The second barrier in draining infected pleural fluid is the high fluid viscosity. Piccolo et al. [6] addressed this in the MIST-2 trial, a randomized trial comparing the use of intrapleural DNase and tissue plasminogen activator ( $t$ PA) with placebo. Only the combination DNase/t-PA was superior to placebo showing significantly shortened hospital stay. It would seem the combination therapy has a synergistic effect as neither t-PA nor DNase alone was superior to placebo.

In this issue of Respiration, Mehta et al. [7] explore an alternative dosing regimen for intrapleural t-PA/DNase, as the appropriate dose for individual patients is not known, nor has dosing frequency been compared in any of the previous studies. The authors essentially replicated the methodology of MIST-2, but replacing the twice-daily regimen with a once-daily regimen with a similar protocol where t-PA was instilled followed $2 \mathrm{~h}$ later by $\mathrm{DN}$ ase. The authors report a very low failure rate when using t-PA/DNase as initial therapy with only $7 \%$ of patients being referred for surgery. There were very few adverse events and the median hospital stay was 13 days [7].

Reported failure rates in the placebo arms of MIST-1 were only $14 \%$ and in MIST-2 only $16 \%$, which raises two important issues regarding the management of pleural infections. The first is whether optimal drain care and irrigation alone can significantly reduce the failure rates when these patients are managed conservatively. Hooper et al. [8] recently compared pleural irrigation with normal saline to standard care in pleural infection. The saline irrigation resulted in a greater reduction in pleural collection volume and fewer referrals for surgery. The same is evident by the low failure rates in the placebo arms of the initial trials with streptokinase and t-PA/DNase. This may be one of the reasons why these studies showed little difference between streptokinase and placebo or even DNase and placebo; however, this was not the case when combining the two therapies, suggesting possible efficacy superior to that of drain care alone.

The second unanswered question is whether to use combination therapy as initial therapy or as a 'rescue' therapy once conservative management has failed. Considering the relatively low failure rates with conservative management mentioned in the studies above, the economic considerations of shorter hospital stay versus higher cost of therapy become relevant. Mehta and colleagues [7] chose to use t-PA/DNase as initial therapy, commenting on cost savings when using daily versus twice-daily t-PA/DNase. The financial implications of a shorter hospital stay, fewer surgical interventions and a considerable reduction in drug dosages might easily offset the cost of starting all patients on t-PA/DNase, but future studies would be required to prove this.

Clearly, further research is needed to best define the optimal dose and frequency of the t-PA/DNase and whether to use t-PA/DNase as initial treatment for all patients with complicated pleural infection or to use as 'rescue' therapy.

\section{References}

1 Koegelenberg CFN, Diacon AH, Bolliger CT: Parapneumonic pleural effusion and empyema. Respiration 2008;75:241-250.

- Tillett WS, Sherry S: The effect in patients of streptococcal fibrinolysin (streptokinase) and streptococcal desoxyribonuclease on fibrinous, purulent, and sanguinous pleural exudations. J Clin Invest 1949;28:173-190.

-3 Diacon AH, Theron J, Schuurmans MM, Van de Wal BW, Bolliger CT: Intrapleural streptokinase for empyema and complicated parapneumonic effusions. Am J Respir Crit Care Med 2004;170:49-53.

4 Maskell NA, Davies CW, Nunn AJ, et al: U.K. controlled trial of intrapleural streptokinase for pleural infection. N Engl J Med 2005;352: 865-874.
5 Diacon AH, Koegelenberg CFN, Bolliger CT: A trial of intrapleural streptokinase. $\mathrm{N}$ Engl J Med 2005;352:2243.

-6 Piccolo F, Popowicz N, Wong D, Lee YC: Intrapleural tissue plasminogen activator and deoxyribonuclease therapy for pleural infection. J Thorac Dis 2015;7:999-1008.

7 Mehta HJ, Biswas A, Penley AM, Cope J, Barnes M, Jantz MA: Management of intrapleural sepsis with once daily use of tissue plasminogen activator and deoxyribonuclease. Respiration 2016;91:101-106.

$\checkmark 8$ Hooper CE, Edey AJ, Wallis A, et al: Pleural irrigation trial (PIT): standard care versus pleural irrigation, a randomised controlled trial in patients with pleural infection. Eur Resp J 2015;46:456-463. 